\title{
Prognostic Value of Neutrophil:Lymphocyte and Platelet:Lymphocyte Ratios for 28-Day Mortality of Patients with AECOPD
}

\section{Zhuanbo Luo \\ Wen Zhang \\ Lina Chen \\ Ning $X u$}

Department of Respiratory Disease, Ningbo First Hospital, Zhejiang, People's Republic of China
Correspondence: Zhuanbo Luo Department of Respiratory Disease, Ningbo First Hospital, 59 Liuting Street, Haishu District, Ningbo, Zhejiang, 3 I5010, People's Republic of China Tel + I58-678|-3738

Email luozhuanbo2929@I63.com
Objective: Acute exacerbation of chronic obstructive pulmonary disease (AECOPD) is the most common cause of hospitalization and death among COPD patients. Clinicians are seeking simple, inexpensive, and easily obtained biomarkers for prognostic evaluation. The aim of this study was to evaluate the association of the neutrophil:lymphocyte ratio (NLR), platelet:lymphocyte ratio (PLR) and lymphocyte:monocyte ratio (LMR) with 28-day mortality and assess the clinical prognostic utility of the NLR, PLR, and LMR in patients with AECOPD.

Methods: A retrospective study was conducted from January 2017 to April 2020 at Ningbo First Hospital. Clinical characteristics, NLR, PLR, LMR, serum levels of CRP, and other data were collected. Relationships between the NLR/PLR/LMR and CRP were evaluated with Pearson's correlation test. Receiver-operating characteristic curves and area under the curve were used to assess the ability of NLR/PLR/LMR to predict 28-day mortality in patients with AECOPD. Optimal cutoff values were determined by the Youden index. Univariate and multivariate logistic regression analysis were used to identify risk factors for 28-day mortality in patients with AECOPD.

Results: By reviewing the medical case records, we collected 533 cases diagnosed with AECOPD for analysis. Death had occurred in 48 (9\%) patients within 28 days. Univariate analysis identified age, smoking history, $\mathrm{FEV}_{1} \%$ predicted, neutrophil count, lymphocyte count, NLR, PLR, CRP and blood-urea nitrogen as being associated with increased 28-day mortality. Multivariate analysis demonstrated that elevated NLR and PLR were significantly associated with death at 28 days. Receiver-operating characteristic analysis showed that the NLR had the highest area under the curve $(0.801)$, with optimal cutoff of 6.74 , sensitivity of $82.54 \%$, and specificity of $71.38 \%$. At a cutoff of 203.6 , the corresponding sensitivity, specificity, and area under the curve of the PLR were $76.86 \%, 65.27 \%$, and 0.75 . The LMR failed to show prognostic significance.

Conclusion: Our results indicated that the NLR and PLR were associated with 28-day mortality in patients with AECOPD. These ratios may serve as prognostic biomarkers for short-term mortality in patients hospitalized with AECOPD.

Keywords: prognostic value, neutrophil to lymphocyte ratio, platelet to lymphocyte ratio, AECOPD, biomarker

\section{Introduction}

Characterized by progressive breathing difficulty and airflow limitation, chronic obstructive pulmonary disease (COPD) is considered one of the main diseases in modern times. ${ }^{1}$ It is estimated that there are around 3 million deaths annually due to 
COPD worldwide. ${ }^{2}$ With the increasing prevalence of smoking in developing countries and aging populations in high-income countries, the prevalence of COPD is expected to rise over the next 40 years, and by 2060 there may be over 5.4 million deaths annually because of COPD and related conditions. ${ }^{3,4}$ In China, COPD is also a major public-health concern. Over the past decade, the overall incidence of COPD in Chinese people older than 40 years has risen from $8.2 \%$ to $13.7 \%{ }^{5,6}$ Due to continued exposure to COPD risk factors, prevalence and burden are projected to increase. Acute exacerbation of COPD (AECOPD) is acute worsening of the patient's respiratory symptoms (increased dyspnea, increased sputum volume, production of purulent sputum), and is the most common cause of hospitalization and death among COPD patients. Accurately assessing the severity and predicting the outcome of AECOPD are of critical importance for clinical management and optimal allocation of limited medical resources. Although the underlying mechanism is still unclear, systemic inflammation has been blamed. Previous studies have suggested that inflammation in AECOPD is amplified in comparison with stable periods, and increased levels of inflammatory markers are associated with lung-function decline. ${ }^{7,8}$ Therefore, circulating biomarkers that reflect the status of inflammation can serve as potential predictors for the prognosis of AECOPD patients.

Blood parameters, including numbers of white blood cells (WBCs), neutrophils, platelets, lymphocytes, and neutrophil:lymphocyte ratio (NLR), platelet:lymphocyte ratio (PLR), lymphocyte:monocyte ratio (LMR), and hypersensitive CRP, are regarded as reliable indicators of systemic inflammation. 9,10 The NLR, PLR, and LMR have been proposed as simple and inexpensive independent predictors in many diseases. The NLR has been proven as a prognostic predictor in many types of malignant tumors, including pancreatic cancer, $^{11}$ esophageal cancer, ${ }^{12}$ metastatic melanoma, ${ }^{13}$ colorectal cancer, ${ }^{14}$ diffuse large B-cell lymphoma, ${ }^{15}$ and non-small cell lung cancer. ${ }^{16}$ In other diseases, elevated NLR has been found to be a marker of poorer outcomes, such as chronic kidney disease, coronary artery disease, appendicitis, systemic lupus erythematosus, and cystic fibrosis. ${ }^{17-21}$ PLR and LMR are other indices of systemic inflammation. Studies have demonstrated the prognostic role of PLR and LMR in many diseases, such as breast cancer, ${ }^{22}$ laryngeal squamous-cell carcinoma, ${ }^{23}$ non-small cell lung cancer, ${ }^{24}$ and thoracolumbar kyphotic deformity. ${ }^{25}$ However, limited data have been presented on the relationship between NLR/PLR/LMR and short-term mortality of hospitalized patients with AECOPD. On that basis, our study aimed to investigate the roles of simple, inexpensive, and easily available inflammatory markers, such as NLR, PLR, and LMR, in predicting 28-day mortality for AECOPD patients.

\section{Methods}

\section{Study Population}

We retrospectively enrolled 533 inpatients with a diagnosis of AECOPD in our hospital from January 2017 to April 2020. Diagnosis owas based on clinical evaluation and pulmonary function tests showing airflow obstruction according to the GOLD criteria (forced expiratory volume in 1 second $\left[\mathrm{FEV}_{1}\right]<80 \%$ predicted, $\mathrm{FEV}_{1} /$ forced vital capacity [FVC] $<70 \%$, and bronchodilatation effect $<12 \%$ ). ${ }^{1}$ AECOPD was defined as altered amount and color of sputum, increased coughing, increased chest tightness, wheezing, pyrexia, decreased performance in daily activities, and/or altered mental status. ${ }^{1}$ Criteria for admission were frequent exacerbation, impaired activities of daily life because of shortness of breath, fever, and/or deterioration in mental status, increased oxygen demand $\left(\mathrm{PaO}_{2}<60 \mathrm{mmHg}, \mathrm{SaO}_{2}<90 \%\right)$, and/or hypercapnia $(>50 \mathrm{mmHg})$, despite optimal treatment. ${ }^{26}$ Patients with hematologic disease, hepatic impairment, active bleeding, blood transfusion in the preceding 3 months, pulmonary embolism in the last month, acute myocardial infarction or cerebrovascular disease, history of myeloproliferative disease, autoimmune diseases, and any other form of cancer were excluded from the study. The Ethics Committee of Ningbo First Hospital approved the study, which was carried out according to the Declaration of Helsinki. All participants provided written informed consent.

\section{Data Collection}

Venous blood was collected twice from each recruited individual. The first test was performed immediately after admission and the second 7-10 days after admission. Demographic information, ie, age, sex, smoking status, and body-mass index (BMI), and blood-count parameters, ie, WBCs, neutrophils, lymphocytes, platelets, monocytes, and CRP were collected from medical records. NLR, PLR, and LMR were all obtained from the same automated blood samples for analysis. Blood-cell analysis was performed on an MEK-8222K automatic hematology analyzer (Nihon Kohden). The other parameters - D-dimer and 
fibrinogen levesl, serum creatinine $(\mathrm{Cr})$, blood-urea nitrogen (BUN), albumin, and lactate dehydrogenase (LDH) were also retrieved from medical records. For arterial blood-gas analyses, blood was drawn from the radial artery while the patients were breathing room air. Spirometry was performed according to American Thoracic Society guidelines. Lung function data, ie, $\mathrm{FEV}_{1}, \mathrm{FVC}, \mathrm{FEV}_{1} / \mathrm{FVC}$, and $\mathrm{FEV}_{1} \%$ predicted, were collected from all subjects.

\section{Statistical Analysis}

All statistical analyses were performed using SPSS 20.0. Patients were divided into two groups (death group and survival group) according to outcome after 28 days' hospitalization. Continuous variables are presented as means \pm SD. Categorical data are presented as frequencies and percentages. Continuous variables were tested for normality using the Kolmogorov-Smirnov test. Differences in continuous variables between groups were determined using Student's $t$-test or nonparametric tests for variables with or without normal distribution, respectively. Paired-sample $t$-tests were used to compare differences in parameters at the time of admission and 7-10 days later. Categorical data were compared using the $\mathrm{X}^{2}$ tests. Relationships between different parameters were evaluated by Pearson's correlation test. Receiver- operating characteristic (ROC) curves were constructed for NLR/PLR/LMR/CRP. Area under the ROC curve (with $95 \% \mathrm{CI}$ ), sensitivity, and specificity were calculated and compared with one another. Optimal cutoff values were determined by the Youden index. Univariate and multivariate logistic regression analysis was used to identify risk factors of 28-day mortality in patients with AECOPD. Variables from the univariate analysis with $P<0.05$ were evaluated for multivariate binary logistic regression. $P<0.05$ was considered statistically significant.

\section{Results}

\section{General Characteristics of All Subjects}

We reviewed medical case records from January 2017 to April 2020. Finally, 533 patients with AECOPD met our inclusion criteria and were included. A total of 48 patients had died within 28 days ( $9 \%$ ). The patients were divided into a death group (48 cases) and survival group (485 cases). General characteristics and comparison of all parameters are listed in Table 1 . The mean age of all participants was $75.71 \pm 9.92$ years and mean BMI 20.74 \pm 3.55 .
Men accounted for $66.6 \%$, and $338(63.41 \%)$ had a history of smoking.

\section{Parameters of Death and Survival Groups at Admission}

There were no significant differences between the death group and survival group for sex $(P=0.525)$ or BMI $(21.16$ \pm 3.73 vs $19.40 \pm 2.49, P=0.675)$. However, the smoking rate in the death group was significantly higher than the survival group $(P=0.022)$. Mean age of the death group was significantly higher than the survival group (74.76 \pm 9.95 vs $80.87 \pm 8.66, P<0.001)$. The survival group had significantly higher $\mathrm{FEV}_{1} \%$ predicted than the death group (45.72 \pm 8.03 vs $39.23 \pm 5.56, P<0.001$ ), but differences in FEV1 and $\mathrm{FEV}_{1} / \mathrm{FVC}$ between the two groups were not significant. These results are listed in Table 1.

Compared to the survival group, the death group had significantly elevated WBCs $(12.14 \pm 3.91$ vs $8.76 \pm 4.23$, $P<0.001)$, neutrophils ( $10.60 \pm 3.97$ vs $6.81 \pm 5.68, P<0.001)$, NLR $(15.12 \pm 12.99$ vs $8.51 \pm 6.08, P<0.001)$ and PLR $(284.70$ \pm 131.73 vs $211.92 \pm 89.57, P<0.001$ ) over the survival group. CRP levels in the death group were also higher than the survival group $(64.65 \pm 28.68$ vs $43.79 \pm 19.91, P<0.001)$. The death group also had significantly higher $\mathrm{PaCO}_{2}(63.03 \pm 17.36$ vs 51.58 $\pm 14.70, P<0.001)$, D-dimer (933.27 \pm 238.88 vs 350.56 $\pm 105.07, \quad P<0.001), \quad$ Cr $\quad(101.00 \pm 66.64$ vs $77.46 \pm 47.51$, $P=0.005)$, BUN $(10.63 \pm 1.99$ vs $7.21 \pm 1.81, P=0.007)$ and LDH (291.23 \pm 88.92 vs $212.64 \pm 84.14, P=0.001)$. However, the death group had markedly reduced $\mathrm{PaO}_{2}(65.94 \pm 9.43$ vs $70.9 \pm 10.21, P=0.002)$ and lymphocytes $(0.86 \pm 0.67$ vs 1.22 $\pm 0.75, P<0.001)$ compared to the survival group. Differences in $\mathrm{pH}$, LMR, platelets, monocytes, fibrinogen, and albumin were not significant. These results are presented in Table 1. NLR and PLR correlated positively with CRP $(r=0.472$, $P<0.001 ; r=0.233, P=0.002$ ), whereas no correlation was observed between LMR and CRP $(r=-0.135, P=0.445)$.

We analyzed the subgroup with elevated $\mathrm{PaCO}_{2}$ $(\geq 50 \mathrm{mmHg})$ at admission. We found 265 patients with elevated $\mathrm{PaCO}_{2}: 227$ in the survival group and 38 in the death group. NLR and PLR in nonsurvivors were markedly higher than in survivors $(18.42 \pm 15.05$ vs $11.16 \pm 7.38$, $P<0.001 ; 294.34 \pm 134.63$ vs $219.53 \pm 96.74, P<0.001)$. We also analyzed the subgroup with reduced $\mathrm{PaO}_{2}$ $(\leq 60 \mathrm{mmHg}$ ) at admission. We found 212 patients with reduced $\mathrm{PaO}_{2}: 183$ in the survival group and 29 in the death group. The death group showed significantly lower $\mathrm{PaO}_{2}(52.74 \pm 21.15$ vs $58.76 \pm 27.88, P<0.05)$ than the 
Table I Comparison of general characteristics between death group and survival group

\begin{tabular}{|c|c|c|c|c|}
\hline Clinical characteristic & Overall $(n=533)$ & Survival group $(n=485)$ & Death group $(n=48)$ & $\mathbf{p}$ \\
\hline Age (years) & $75.7 I \pm 9.92$ & $74.76 \pm 9.95$ & $80.87 \pm 8.66$ & $<0.001$ \\
\hline \multicolumn{5}{|l|}{ Sex, n (\%) } \\
\hline Male & $355(66.60 \%)$ & $325(67.01 \%)$ & $30(62.50 \%)$ & 0.525 \\
\hline Female & $178(33.40 \%)$ & $160(32.99 \%)$ & $18(37.50 \%)$ & \\
\hline \multicolumn{5}{|l|}{ Smoking history, n (\%) } \\
\hline Current/ever-smoker & $338(63.41 \%)$ & $300(61.86 \%)$ & $38(79.17 \%)$ & 0.022 \\
\hline Never-smoker & 195(36.59\%) & $185(38.14 \%)$ & $10(20.83 \%)$ & \\
\hline BMI $\left(\mathrm{kg} / \mathrm{m}^{2}\right)$ & $20.74 \pm 3.55$ & $21.16 \pm 3.73$ & $19.40 \pm 2.49$ & 0.675 \\
\hline \multicolumn{5}{|l|}{ Pulmonary function } \\
\hline $\mathrm{FEV}_{\text {। }}(\mathrm{L})$ & $0.98 \pm 0.43$ & $1.02 \pm 0.55$ & $0.95 \pm 0.49$ & 0.245 \\
\hline $\mathrm{FEV}_{\mathrm{I}} / \mathrm{FVC}$ & $54.84 \pm 9.93$ & $55.13 \pm 10.32$ & $52.17 \pm 8.85$ & 0.334 \\
\hline $\mathrm{FEV}, \%$ predicted & $43.09 \pm 7.61$ & $45.72 \pm 8.03$ & $39.23 \pm 5.56$ & $<0.001$ \\
\hline pH & $7.40 \pm 0.08$ & $7.42 \pm 0.09$ & $7.37 \pm 0.06$ & 0.073 \\
\hline $\mathrm{PaCO}_{2}(\mathrm{mmHg})$ & $53.14 \pm 16.73$ & $51.58 \pm 14.70$ & $63.03 \pm 17.36$ & $<0.001$ \\
\hline $\mathrm{PaO}_{2}(\mathbf{m m H g})$ & $70.0 \pm 10.26$ & $70.90 \pm 10.21$ & $65.94 \pm 9.43$ & 0.002 \\
\hline WBCs $\left(\times 10^{9} / \mathrm{L}\right)$ & $9.35 \pm 4.35$ & $8.76 \pm 4.23$ & $|2.14 \pm 3.9|$ & $<0.001$ \\
\hline Neutrophils $\left(\times 10^{9} / \mathrm{L}\right)$ & $7.58 \pm 5.49$ & $6.8 I \pm 5.68$ & $10.60 \pm 3.97$ & $<0.001$ \\
\hline Lymphocytes $\left(\times 10^{9} / \mathrm{L}\right)$ & $1.12 \pm 0.67$ & $1.22 \pm 0.75$ & $0.86 \pm 0.67$ & $<0.001$ \\
\hline Platelets $\left(\times 10^{9} / \mathrm{L}\right)$ & $211.20 \pm 87.65$ & $213.97 \pm 81.95$ & $200.03 \pm 109.21$ & 0.407 \\
\hline Monocytes $\left(\times 10^{9} / \mathrm{L}\right)$ & $0.64 \pm 0.37$ & $0.64 \pm 0.33$ & $0.62 \pm 0.52$ & 0.663 \\
\hline NLR & $9.02 \pm 6.54$ & $8.5 I \pm 6.08$ & $15.12 \pm 12.99$ & $<0.001$ \\
\hline PLR & $220.99 \pm 95.32$ & $211.92 \pm 89.57$ & $284.70 \pm 131.73$ & $<0.001$ \\
\hline LMR & $2.20 \pm 1.75$ & $2.26 \pm 1.79$ & $1.62 \pm 1.19$ & 0.061 \\
\hline CRP & $45.67 \pm 21.66$ & $43.79 \pm 19.91$ & $64.65 \pm 28.68$ & $<0.001$ \\
\hline D-dimer (ng/mL) & $461.11 \pm 138.99$ & $350.56 \pm 105.07$ & $933.27 \pm 238.88$ & $<0.001$ \\
\hline Fibrinogen (g/L) & $4.09 \pm 2.16$ & $4.15 \pm 2.28$ & $3.85 \pm 1.56$ & 0.386 \\
\hline $\mathrm{Cr}(\mu \mathrm{mol} / \mathrm{L})$ & $81.92 \pm 52.35$ & $77.46 \pm 47.51$ & $101.00 \pm 66.64$ & 0.005 \\
\hline BUN (mmol/L) & $7.86 \pm 1.94$ & $7.21 \pm 1.81$ & $10.63 \pm 1.99$ & 0.007 \\
\hline Albumin (g/L) & $35.11 \pm 7.09$ & $34.56 \pm 5.42$ & $37.42 \pm 10.65$ & 0.645 \\
\hline LDH (U/L) & $227.55 \pm 85.68$ & $212.64 \pm 84.14$ & $291.23 \pm 88.92$ & 0.001 \\
\hline
\end{tabular}

Abbreviations: NLR, neutrophil:lymphocyte ratio; PLR, platelet:lymphocyte ratio; LMR, lymphocyte:monocyte ratio; Cr, creatinine; BUN, blood-urea nitrogen; LDH, lactate dehydrogenase; $\mathrm{FEV}_{1}$, forced expiratory volume in I second; FVC, forced vital capacity.

survival group. Correspondingly, NLR and PLR in nonsurvivors were remarkably higher than in survivors $(17.38$ \pm 10.30 vs $10.66 \pm 5.72, P<0.001 ; 287.56 \pm 121.18$ vs 214.43 $\pm 91.24, P<0.001)$. These results indicated that NLR and PLR can discriminate subgroups with poor prognoses, even when they have elevated or reduced $\mathrm{PaO}_{2}$.
Parameters at Admission Versus 7-10 Days after Hospitalization in Death and Survival Groups

In the death group, compared with admission, WBCs (15.91 \pm 5.65 vs $12.14 \pm 3.91, P<0.001)$, neutrophils (13.45 \pm 4.50 vs $10.60 \pm 3.97, P=0.003)$, NLR $(18.98 \pm 24.34$ vs 
$15.12 \pm 12.99, P<0.001)$, PLR $(302.26 \pm 144.24$ vs 284.70 $\pm 131.73, \quad P<0.001)$, and CRP $(88.76 \pm 34.91$ vs 64.65 $\pm 28.68, P=0.02$ ) were significantly elevated at $7-10$ days. Lymphocytes $(0.69 \pm 0.43$ vs $0.86 \pm 0.67, P=0.001)$ and $\mathrm{PaO} 2(59.56 \pm 7.67$ vs $65.94 \pm 9.43, \mathrm{P}=0.03)$ were significantly lower at 7-10 days than at admission. Other parameters, including $\mathrm{PaCO}_{2}$ and $\mathrm{LDH}$, were elevated significantly $(P<0.05)$ at $7-10$ days. There were no significant differences for D-dimer, Cr, or BUN.

In the survival group, after 7-10 days' treatment, the NLR $(6.77 \pm 4.87$ vs $8.51 \pm 6.08, P<0.001)$, PLR (193.74 \pm 77.34 vs $211.92 \pm 89.57, P=0.02)$, and CRP $(21.63 \pm 9.39$ vs $43.79 \pm 19.91, P<0.001)$ had decreased significantly compared to admission, but the lymphocytes had elevated significantly $(2.08 \pm 0.89$ vs $1.22 \pm 0.75, P=0.005)$. WBC and neutrophils had reduced at 7-10 days, but the differences were not significant. D-dimer, $\mathrm{Cr}$, and BUN had decreased significantly $(P<0.05)$ and $\mathrm{PaO}_{2}$ elevated significantly $(P=0.002)$ after treatment. There were no significant differences in $\mathrm{PaCO}_{2}$ and $\mathrm{LDH}$. Results wae presented in the Table 2 .

\section{Univariate and Multivariate Logistic Regression of Risk Factors Associated with 28-Day Mortality}

On univariate analysis, factors significantly correlating with increased 28-day mortality were age (OR 1.07, 95\% CI 1.03-1.27; $P=0.001$ ), smoking history (OR 1.27, 95\% CI $0.56-3.04 ; P=0.015$ ), $\mathrm{FEV}_{1} \%$ predicted (OR $0.96,95 \%$ CI $0.95-0.99 ; P=0.004$ ), neutrophils (OR 3.98, 95\% CI 1.91-7.06; $P<0.001$ ), lymphocytes (OR 1.79, 95\% CI $2.37-11.69 ; P=0.007$ ), NLR (OR $4.09,95 \%$ CI $1.03-$ 9.14; $P<0.001$ ), PLR (OR 4.03, 95\% CI 1.33-11.01; $P<0.001$ ), CRP (OR 3.03, 95\% CI 1.84-3.05; $P<0.001$ ), and BUN (OR 1.87, 95\% CI 0.99-2.03; $P=0.018$; Table 3). There was no significant difference in $\mathrm{PaCO}_{2}, \mathrm{PaO}_{2}$, D-dimer, $\mathrm{Cr}$, or LDH $(P>0.05$, Table 3$)$. Multivariate logistic regression analysis of the significant variables demonstrated that elevated NLR (OR 3.87, 95\% CI 1.2910.30; $P<0.001$ ) and PLR (OR 3.45, 95\% CI 1.43-12.62; $P=0.005)$ were also significantly associated with 28 -day mortality. Smoking, decreased $\mathrm{FEV}_{1} \%$ predicted, elevated neutrophils, and elevated CRP were also associated with increased adverse outcomes. These data are presented in Table 4.

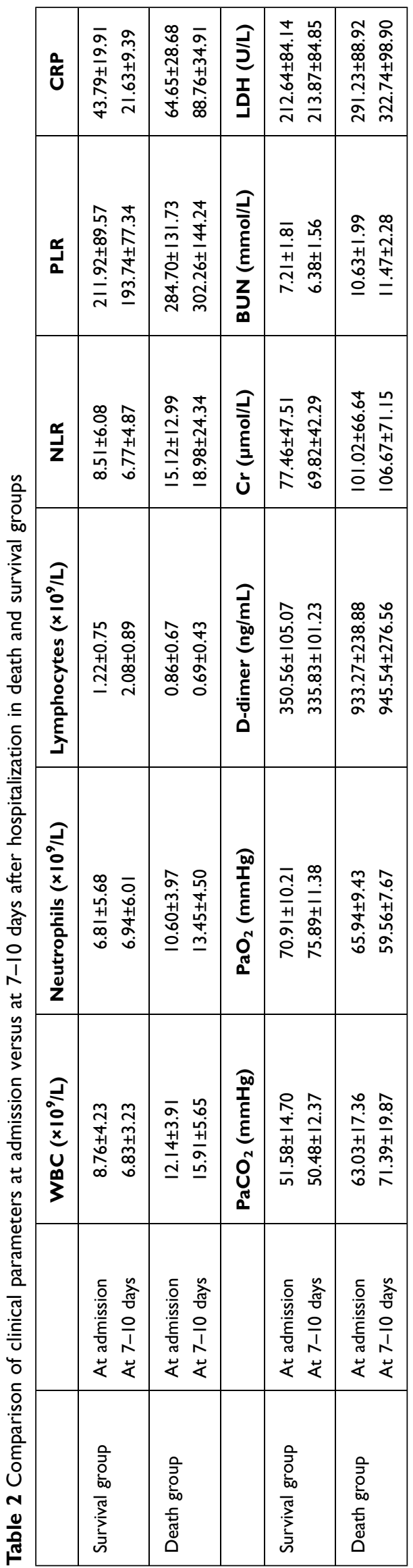


Table 3 Univariate logistic regression analysis of risk factors associated with 28-day mortality for AECOPD patients

\begin{tabular}{|c|c|c|c|c|c|c|}
\hline Parameter & $\boldsymbol{\beta}$ & SE & Wald $x^{2}$ & $\mathbf{P}$ & OR & $95 \% \mathrm{Cl}$ \\
\hline Age (years) & 0.07 & 0.02 & 11.39 & 0.001 & 1.07 & $1.03-1.27$ \\
\hline Smoking history & 0.37 & 0.33 & 1.04 & 0.015 & 1.27 & $0.56-3.04$ \\
\hline $\mathrm{FEV}, \%$ predicted & $-0.4 I$ & 0.15 & 14.78 & 0.004 & 0.96 & $0.95-0.99$ \\
\hline $\mathrm{PaCO}_{2}(\mathrm{mmHg})$ & 1.06 & 0.21 & 31.14 & 0.065 & 1.36 & $1.04-2.28$ \\
\hline $\mathrm{PaO}_{2}(\mathrm{mmHg})$ & 0.95 & 0.32 & 9.15 & 0.072 & 1.95 & $1.92-2.98$ \\
\hline WBCs $\left(\times 10^{9} / \mathrm{L}\right)$ & 0.90 & 0.25 & 2.18 & 0.341 & $0.4 I$ & $0.29-2.21$ \\
\hline Neutrophils $\left(\times 10^{9} / \mathrm{L}\right)$ & 1.02 & 0.44 & 11.20 & $<0.001$ & 3.98 & $1.91-7.06$ \\
\hline Lymphocytes $\left(\times 10^{9} / \mathrm{L}\right)$ & 1.73 & 0.38 & 21.36 & 0.007 & 1.79 & $2.37-11.69$ \\
\hline NLR & 1.08 & 0.43 & 59.76 & $<0.001$ & 4.09 & $1.03-9.14$ \\
\hline PLR & 2.00 & 0.60 & 37.59 & $<0.001$ & 4.03 & $1.33-11.01$ \\
\hline CRP & 1.03 & 0.61 & 8.16 & $<0.001$ & 3.03 & I.84-3.05 \\
\hline D-dimer (ng/mL) & 1.53 & 0.51 & 32.62 & 0.105 & 1.08 & $0.87-1.54$ \\
\hline $\mathrm{Cr}(\mu \mathrm{mol} / \mathrm{L})$ & 1.69 & 0.16 & 34.09 & 0.073 & 1.01 & $0.47-2.01$ \\
\hline BUN (mmol/L) & 0.71 & 0.33 & 21.77 & 0.018 & 1.87 & $0.99-2.03$ \\
\hline LDH (U/L) & 0.82 & 0.42 & 4.84 & 0.360 & 1.72 & $0.98-1.97$ \\
\hline
\end{tabular}

\section{ROC-Curve Analysis}

We conducted post hoc analyses to determine the prognostic utility of the NLR, PLR, LMR, and CRP by calculating sensitivity and specificity using the ROC curves. The NLR had the highest AUC $(0.801,95 \%$ CI $0.753-$ 0.889 ), with optimal cutoff of 6.74 , sensitivity of $82.54 \%$, and specificity of $71.38 \%$. At a cutoff of 203.60, the corresponding sensitivity, specificity, and AUC of the PLR were $76.86 \%, 65.27 \%$, and 0.750 . At a cutoff of 56.65, the sensitivity, specificity, and AUC of CRP were $73.86 \%, 61.27 \%$, and 0.740 . However, the AUC of the LMR was only 0.368 . Next, we investigated the predictive value of different biomarker combinations: combining markers increased predictive sensitivity, with the best AUC 0.857 , sensitivity of $86.54 \%$, and specificity of $70.85 \%$ obtained using a combination of NLR, PLR, and CRP. Figure 1 shows ROC curves for the NLR, PLR, and CRP and combination of three markers.

\section{Discussion}

AECOPD represents episodes of deterioration of respiratory symptoms that reflect worsening of underlying chronic airway inflammation. ${ }^{27}$ It is considered the leading cause of hospitalization, and contributes significantly to mortality among patients with COPD. ${ }^{28}$ Identifying reliable and simple biomarkers that can correctly assess mortality risk during AECOPD hospitalization is of great importance in clinical practice. In this study, we found that NLR and PLR in the death group were significantly higher than the survival group, and these ratios were positively correlated with CRP - a traditional systemic inflammation biomarker - in patients with AECOPD. Multivariate binary logistic regression analysis revealed that increased NLR and PLR were independent risk factors of 28-day mortality in AECOPD patients. ROC analysis showed that the AUC of the NLR and PLR for predicting 28-day mortality was 0.801 and 0.750 , indicating predictive accuracy. Furthermore, the NLR and PLR had significantly elevated in nonsurvivors at 7-10 days over admission, indicating that they had increased as the patient's condition worsened, thereby providing a reference for clinicians. However, the LMR did not show a significant relationship with short-term death in AECOPD.

It has been observed that systemic inflammatory markers will increase in COPD patients, and during exacerbations, the severity of inflammation is significantly enhanced. ${ }^{29}$ There have been several studies showing that such inflammatory 
Table 4 Multivariate logistic regression analysis of risk factors associated with 28-day mortality for AECOPD patients

\begin{tabular}{|l|c|c|c|c|c|c|}
\hline Parameter & $\boldsymbol{\beta}$ & SE & Wald $\chi^{2}$ & $\mathbf{P}$ & OR & 95\% CI \\
\hline Age (years) & 0.12 & 0.08 & 10.34 & 0.672 & 0.79 & $0.65-1.44$ \\
\hline Smoking history & 0.88 & 0.74 & 17.38 & 0.026 & 2.02 & $1.78-5.13$ \\
\hline FEVI\% predicted & -0.34 & 0.32 & 15.28 & 0.034 & 0.97 & $0.96-0.99$ \\
Neutrophils (×109/L) & 1.21 & 0.54 & 12.34 & 0.002 & 1.78 & $1.35-6.31$ \\
\hline Lymphocytes (×109/L) & 1.64 & 0.33 & 18.79 & 0.083 & 1.04 & $1.86-10.37$ \\
\hline NLR & 1.22 & 0.52 & 52.28 & $<0.001$ & 3.87 & $1.29-10.30$ \\
\hline PLR & 2.15 & 0.58 & 33.89 & 0.005 & 3.45 & $1.43-12.62$ \\
\hline CRP & 1.14 & 0.38 & 7.73 & $<0.001$ & 2.58 & $1.59-4.47$ \\
\hline BUN (mmol/L) & 0.77 & 0.41 & 17.76 & 0.076 & 0.88 & $0.76-2.78$ \\
\hline
\end{tabular}

markers as CRP, IL-6, IL-8, and TNF- $\alpha$ increase in patients with COPD and even those with stable COPD compared to healthy individuals, and that increased levels of these markers are associated with acute exacerbation and mortality. ${ }^{30,31} \mathrm{CRP}$ is one of the most commonly used inflammatory markers in clinical practice. In this study, we also found that serum CRP levels significantly increased in the death group compared to the survival group. As an emerging inflammatory indicator, the

\section{ROC curve}

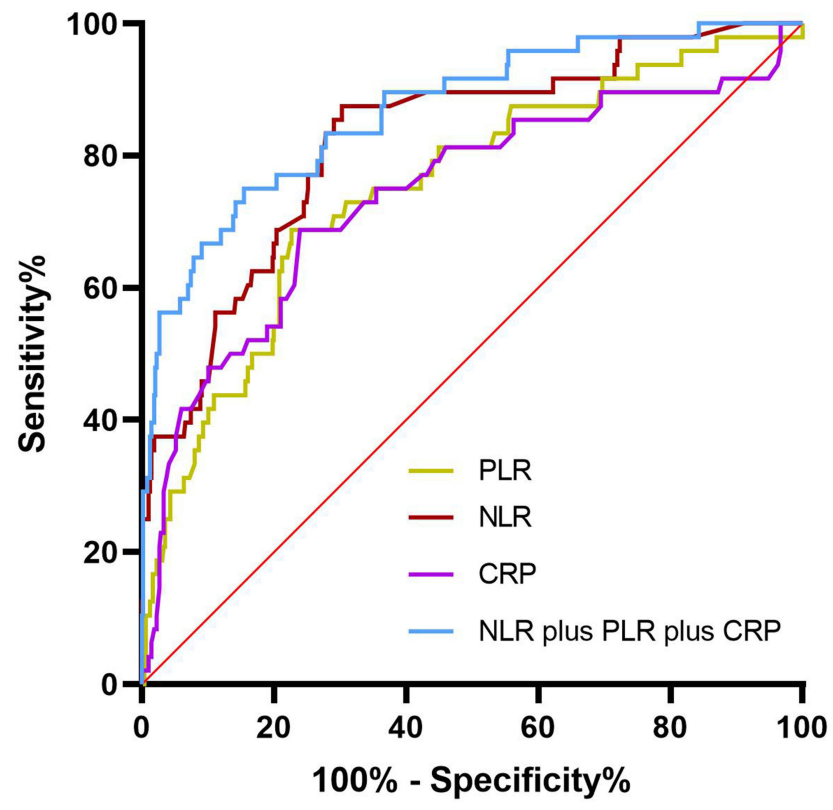

Figure I Receiver-operating characteristic (ROC) curves of NLR, PLR, and CRP, singly and combined, for predicting 28-day mortality of patients with acute exacerbation of chronic obstructive pulmonary disease. ROC curves for single predictors had areas of 0.801 (NLR), 0.750 (PLR), and 0.740 (CRP) . Combined predictors of NLR, PLR, and CRP had the best AUC of 0.857 .
NLR plays multiple roles in acute exacerbations of COPD and is helpful in the clinical management of patients with AECOPD. The NLR has been demonstrated to be as useful as CRP in the evaluation of elevated inflammation in AECOPD.$^{32}$ Lee et $\mathrm{al}^{33}$ found that the NLR was significantly elevated in patients with AECOPD over those with stable COPD. Kurtipek et al demonstrated that as NLR increased, airway narrowing increased, indicating the NLR was a useful marker in early identification for further COPD exacerbations. ${ }^{34}$ In another study, NLR $>4.5$ was found to be an independent risk factor of readmission due to AECOPD within a month. ${ }^{35}$ Lee et $\mathrm{al}^{36}$ found that the NLR correlated with the severity of airflow limitation, and that it can be used as a predictor for exacerbations during 1-year follow-up. Recently, two more studies demonstrated that the NLR has a prognostic role for long-term mortality in COPD patients. ${ }^{37,38}$ In our study, we found that NLR was higher in nonsurvivors than survivors with AECOPD, and NLR correlated positively with CRP. At an optimal cutoff of 6.74 , the sensitivity and specificity of the NLR in predicting 28-day mortality were $82.54 \%$ and $71.38 \%$, respectively, with an AUC of 0.801 . The accuracy of this evaluation was much better than that of CRP (AUC 0.740) and PLR (AUC 0.750). Therefore, our present results demonstrate that the NLR has moderate diagnostic ability for short-term prognosis and is useful as a prognostic biomarker in AECOPD patients.

The PLR, another inflammatory biomarker, has been extensively evaluated as a surrogate marker of inflammation in many diseases. In COPD, two studies with relatively small samples reported that PLR was significantly elevated in AECOPD patients compared to stable COPD patients, but neither specifically explored the association between elevated 
PLR and adverse clinical outcomes. In this study, we evaluated the utility of the PLR to predict short-term mortality in hospitalized patients with AECOPD. The results showed that the PLR was significantly higher in the death group than the survival group and correlated positively with CRP. At a cutoff of 203.60, the sensitivity and specificity of the PLR were $76.86 \%$ and $65.27 \%$, respectively, with an AUC of 0.75 , indicating that it may also be a useful biomarker for identifying AECOPD patients at increased risk of adverse outcomes and providing prognostic guidance to clinicians. We found that the combination of NLR and PLR with CRP increased predictive sensitivity for short-term mortality in patients with AECOPD, with best AUC of 0.857 , sensitivity of $86.54 \%$, and specificity of $70.85 \%$. Therefore, we suggest combining the NLR and PLR with traditional inflammatory markers, such as CRP, to improve the accuracy of prediction.

Our study is a novel contribution to the growing scientific body of knowledge about the prognostic utility of the NLR and PLR in chronic inflammatory disorders, such as COPD. Our results further suggest that the NLR and PLR are simple and useful biomarkers for predicting short-term mortality in hospitalized patients with AECOPD and are available to and affordable for each patient with AECOPD. The clinical utility of the NLR and PLR may be superior to that of other markers, which may need specific equipment or reagents. Monitoring changes in NLR and PLR can help clinicians in early detection of patients with AECOPD who are at high risk of short-term death. However, there were certain limitations in our study. First, this was a retrospective study. There may have been some selection bias. Second, it was limited by using a single medical center for data. Finally, the present results were from the short-term follow-up. Future studies should focus on long-term outcomes. We hope to make further efforts to design a prospective study with multiple centers and different ethnic groups to explore the application values of the NLR and PLR in patients with AECOPD.

\section{Conclusion}

The present study suggested that the NLR and PLR - as rapid, inexpensive, and easily obtained routine blood markers - were associated with short-term adverse outcomes in patients with AECOPD. Elevated NLR and PLR predicted increased risk of 28-day mortality in patients with AECOPD. They may be independent prognostic biomarkers. However, the LMR failed to show prognostic significance. Patients hospitalized for AECOPD may benefit from regular clinical surveillance for NLR and PLR.

\section{Abbreviations}

AECOPD, acute exacerbation of chronic obstructive pulmonary disease; NLR, neutrophil:lymphocyte ratio; PLR, platelet:lymphocyte ratio; LMR, lymphocyte:monocyte ratio; $\mathrm{Cr}$, creatinine; BUN, blood-urea nitrogen; LDH, lactate dehydrogenase; $\mathrm{FEV}_{1}$, forced expiratory volume in 1 second; ROC, receiver-operating characteristic.

\section{Data Sharing Statement}

The data sets used and analyzed during the current study are available from the corresponding author on reasonable request.

\section{Ethics Approval and Consent to Participate}

The Ethics Committee of Ningbo First Hospital approved this study in November 2020 (2020-R63).

\section{Funding}

This study was funded by the TCM (traditional Chinese medicine) Science and Technology Plan of Zhejiang province (2020ZB218).

\section{Disclosure}

The authors declare that they have no competing interests.

\section{References}

1. Chronic Obstructive Pulmonary Disease. Global strategy for the diagnosis, management, and prevention of chronic obstructive pulmonary disease(2020report); 2020. Available from: https://goldcopd.org/wpcontent/uploads/2019/12/GOLD-2020-FINAL-ver1.2-03Dec19_ WMV.pdf. Accessed May 26, 2021.

2. Burgel PR, Nesme-Meyer P, Chanez P, et al. Cough and sputum production are associated with frequent exacerbations and hospitalizations in COPD subjects. Chest. 2009;135:975-982. doi:10.1378/ chest.08-2062

3. Jolliffe DA, Greenberg L, Hooper RL, et al. Vitamin D to prevent exacerbations of COPD: systematic review and meta-analysis of individual participant data from randomised controlled trials. Thorax. 2019;74:337-345. doi:10.1136/thoraxjnl-2018-212092

4. Martinez FJ, Han MK, Flaherty K, Curtis J. Role of infection and antimicrobial therapy in acute exacerbations of chronic obstructive pulmonary disease. Expert Rev Anti Infect Ther. 2006;4:101-124. doi:10.1586/14787210.4.1.101

5. Zhong N, Wang C, Yao W, et al. Prevalence of chronic obstructive pulmonary disease in China: a large, population-based survey. Am J Respir Crit Care Med. 2007;176:753-760. doi:10.1164/rccm.200612-1749OC

6. Wang C, Xu J, Yang L, et al. Prevalence and risk factors of chronic obstructive pulmonary disease in China (the China Pulmonary Health [CPH] study): a national cross-sectional study. Lancet. 2018;391:1706-1717. doi:10.1016/S0140-6736(18)30841-9

7. Peng Z, Zhang W, Qiao J, He B. Melatonin attenuates airway inflammation via SIRT1 dependent inhibition of NLRP3 inflammasome and IL-1beta in rats with COPD. Int Immunopharmacol. 2018;62:23-28. doi:10.1016/j.intimp.2018.06.033 
8. Cheng Q, Fang L, Feng D, et al. Memantine ameliorates pulmonary inflammation in a mice model of COPD induced by cigarette smoke combined with LPS. Biomed Pharmacother. 2019;109:2005-2013. doi:10.1016/j.biopha.2018.11.002

9. Bozan N, Alpayci M, Aslan M, et al. Mean platelet volume, red cell distribution width, platelet-to-lymphocyte and neutrophil-to-lymphocyte ratios in patients with ankylosing spondylitis and their relationships with high-frequency hearing thresholds. Eur Arch Otorhinolaryngol. 2016;273:3663-3672. doi:10.1007/s00405-016-3980-y

10. Mercan R, Bitik B, Tufan A, et al. The association between neutrophil/lymphocyte ratio and disease activity in rheumatoid arthritis and ankylosing spondylitis. $J$ Clin Lab Anal. 2016;30:597-601. doi:10.1002/jcla.21908

11. Yang JJ, Hu ZG, Shi WX, Deng T, He SQ, Yuan SG. Prognostic significance of neutrophil to lymphocyte ratio in pancreatic cancer: a meta-analysis. World J Gastroenterol. 2015;21:2807-2815. doi:10.3748/wjg.v21.i9.2807

12. Yoo EJ, Park JC, Kim EH, et al. Prognostic value of neutrophil-tolymphocyte ratio in patients treated with concurrent chemoradiotherapy for locally advanced oesophageal cancer. Dig Liver Dis. 2014;46:846-853. doi:10.1016/j.dld.2014.05.009

13. Cananzi FC, Dalgleish A, Mudan S. Surgical management of intraabdominal metastases from melanoma: role of the neutrophil to lymphocyte ratio as a potential prognostic factor. World J Surg. 2014;38:1542-1550. doi:10.1007/s00268-013-2418-6

14. Azab B, Mohammad F, Shah N, et al. The value of the pretreatment neutrophil lymphocyte ratio vs. platelet lymphocyte ratio in predicting the long-term survival in colorectal cancer. Cancer Biomark. 2014;14:303-312. doi:10.3233/CBM-140416

15. Troppan K, Deutsch A, Gerger A, et al. The derived neutrophil to lymphocyte ratio is an independent prognostic factor in patients with diffuse large B-cell lymphoma. Br J Cancer. 2014;110:369-374. doi:10.1038/bjc.2013.763

16. Zhang H, Xia H, Zhang L, Zhang B, Yue D, Wang C. Clinical significance of preoperative neutrophil-lymphocyte vs platelet-lymphocyte ratio in primary operable patients with non-small cell lung cancer. $\mathrm{Am}$ J Surg. 2015;210:526-535. doi:10.1016/j.amjsurg.2015.03.022

17. Kocyigit I, Eroglu E, Unal A, et al. Role of neutrophil/lymphocyte ratio in prediction of disease progression in patients with stage-4 chronic kidney disease. J Nephrol. 2013;26:358-365. doi:10.5301/ jn.5000152

18. Poludasu S, Cavusoglu E, Khan W, Marmur JD. Neutrophil to lymphocyte ratio as a predictor of long-term mortality in African Americans undergoing percutaneous coronary intervention. Clin Cardiol. 2009;32:E6-E10. doi:10.1002/clc.20503

19. Hajibandeh S, Hajibandeh S, Hobbs N, Mansour M. Neutrophil-tolymphocyte ratio predicts acute appendicitis and distinguishes between complicated and uncomplicated appendicitis: a systematic review and meta-analysis. Am J Surg. 2020;219:154-163. doi:10.1016/j.amjsurg.2019.04.018

20. Li L, Xia Y, Chen C, Cheng P, Peng C. Neutrophil-lymphocyte ratio in systemic lupus erythematosus disease: a retrospective study. Int $J$ Clin Exp Med. 2015;8:11026-11031.

21. O'Brien CE, Price ET, Hartl D. The blood neutrophil to lymphocyte ratio correlates with clinical status in children with cystic fibrosis: a retrospective study. PLoS One. 2013;8:e77420. doi:10.1371/journal. pone. 0077420

22. Koh $\mathrm{CH}$, Bhoo-Pathy $\mathrm{N}, \mathrm{Ng} \mathrm{KL}$, et al. Utility of pre-treatment neutrophil-lymphocyte ratio and platelet-lymphocyte ratio as prognostic factors in breast cancer. Br J Cancer. 2015;113:150-158. doi:10.1038/bjc.2015.183
23. Chen H, Song S, Zhang L, Dong W, Chen X, Zhou H. Preoperative platelet-lymphocyte ratio predicts recurrence of laryngeal squamous cell carcinoma. Future Oncol. 2020;16:209-217. doi:10.2217/fon2019-0527

24. Zhao QT, Yuan Z, Zhang H, et al. Prognostic role of platelet to lymphocyte ratio in non-small cell lung cancers: a meta-analysis including 3720 patients. Int $J$ Cancer. 2016;139:164-170. doi:10.1002/ijc.30060

25. Zhuang J, Huang Y, Liang G. Clinical significance of the monocyte: lymphocyteratio for ankylosing spondylitis patients with thoracolumbar kyphotic deformities. J Int Med Res. 2020;48:300060519893167. doi:10.1177/0300060519893167

26. Celli BR, MacNee W, Force AET. Standards for the diagnosis and treatment of patients with COPD: a summary of the ATS/ERS position paper. Eur Respir J. 2004;23:932-946. doi:10.1183/ 09031936.04.00014304

27. Papi A, Luppi F, Franco F, Fabbri LM. Pathophysiology of exacerbations of chronic obstructive pulmonary disease. Proc Am Thorac Soc. 2006;3:245-251. doi:10.1513/pats.200512-125SF

28. Lima FV, Yen TY, Patel JK. Trends in in-hospital outcomes among adults hospitalized with exacerbation of chronic obstructive pulmonary disease. COPD. 2015;12:636-642. doi:10.3109/15412555.2015.1020151

29. Gan WQ, Man SF, Senthilselvan A, Sin DD. Association between chronic obstructive pulmonary disease and systemic inflammation: a systematic review and a meta-analysis. Thorax. 2004;59:574-580. doi:10.1136/thx.2003.019588

30. Thomsen M, Dahl M, Lange P, Vestbo J, Nordestgaard BG. Inflammatory biomarkers and comorbidities in chronic obstructive pulmonary disease. Am J Respir Crit Care Med. 2012;186:982-988. doi:10.1164/rccm.201206-1113OC

31. Agusti A, Edwards LD, Rennard SI, et al. Persistent systemic inflammation is associated with poor clinical outcomes in COPD: a novel phenotype. PLoS One. 2012;7:e37483. doi:10.1371/journal.pone.0037483

32. Taylan M, Demir M, Kaya H, et al. Alterations of the neutrophillymphocyte ratio during the period of stable and acute exacerbation of chronic obstructive pulmonary disease patients. Clin Respir J. 2017;11:311-317. doi:10.1111/crj.12336

33. Terradas R, Grau S, Blanch J, et al. Eosinophil count and neutrophillymphocyte count ratio as prognostic markers in patients with bacteremia: a retrospective cohort study. PLoS One. 2012;7:e42860. doi:10.1371/journal.pone.0042860

34. Kurtipek E, Bekci TT, Kesli R, Sami SS, Terzi Y. The role of neutrophil-lymphocyte ratio and platelet-lymphocyte ratio in exacerbation of chronic obstructive pulmonary disease. J Pak Med Assoc. 2015;65:1283-1287.

35. Coban Agca M, Aksoy E, Duman D, et al. Does eosinophilia and neutrophil to lymphocyte ratio affect hospital re-admission in cases of COPD exacerbation? Tuberk Toraks. 2017;65:282-290. doi:10.5578/ tt. 57278

36. Lee H, Um SJ, Kim YS, et al. Association of the neutrophil-tolymphocyte ratio with lung function and exacerbations in patients with chronic obstructive pulmonary disease. PLoS One. 2016;11: e0156511. doi:10.1371/journal.pone.0156511

37. Sorensen AK, Holmgaard DB, Mygind LH, Johansen J, Pedersen C. Neutrophil-to-lymphocyte ratio, calprotectin and YKL-40 in patients with chronic obstructive pulmonary disease: correlations and 5-year mortality - a cohort study. J Inflamm (Lond). 2015;12:20. doi:10.1186/s12950-015-0064-5

38. Xiong W, Xu M, Zhao Y, Wu X, Pudasaini B, Liu JM. Can we predict the prognosis of COPD with a routine blood test? Int $J$ Chron Obstruct Pulmon Dis. 2017;12:615-625. doi:10.2147/COPD. $\mathrm{S} 124041$ 


\section{Publish your work in this journal}

The International Journal of General Medicine is an international, peer-reviewed open-access journal that focuses on general and internal medicine, pathogenesis, epidemiology, diagnosis, monitoring and treatment protocols. The journal is characterized by the rapid reporting of reviews, original research and clinical studies across all disease areas. The manuscript management system is completely online and includes a very quick and fair peer-review system, which is all easy to use. Visit http://www.dovepress.com/ testimonials.php to read real quotes from published authors.

Submit your manuscript here: https://www.dovepress.com/international-journal-of-general-medicine-journal 